\title{
EL VALLE DEL HIERRO-LEGAZPI. UN PUEBLO CONVERTIDO EN UN MUSEO VIVO
}

\section{The Valley of Iron-Legazpi. A people become a living museum}

Olatz Conde

Valle del Hierro-Legazpi

Fundación LENBUR, Legazpi (Gipuzkoa)

oconde@lenbur.com

Recepción: Io de enero de 2016

Envío a informantes: is de enero de 2016

Aceptación definitiva: 27 de febrero de 2016

Resumen: Un pueblo del interior de Gipuzkoa recupera y pone en valor su Patrimonio ligado a la historia del Hierro, para convertir la localidad en un Museo Territorio, un museo vivo donde se guardan y se muestran la tradición y la cultura vascas, desde el siglo XI hasta nuestros días: caseríos, ferrerías, fábricas, viviendas obreras, escuelas, etc. Todo ello con un discurso unificado, donde se comprende realmente por qué se transforma la sociedad rural y artesanal en la sociedad industrial.

Palabras Clave: Gipuzkoa; hierro; Museo; caserío; ferrería; escuela franquista; educación; historia; cultura; tradición.

Aвstract: A town in the interior of Gipuzkoa retrieves and adds value to its linked to the history of the Iron Heritage, to turn the town into a Territory Museum, a living museum where it is kept and tradition and Basque culture is shown, since the eleventh century to this day: farmhouses, foundries, factories, workers' housing, schools, etc. All with a unified discourse, which is really understands why the rural and artisan society is transformed into industrial society.

KeY wORDs: Gipuzkoa; iron; Museum; hamlet; ironworks; Franco school; education; history; culture; tradition.

\section{Legazpi-el Valle del Hierro}

$\mathrm{E}$

N el corazón de Gipuzkoa (a 50 km de San Sebastián), en las faldas del Parque Natural de Aizkorri, atravesado de norte a sur por el río Ur-ola (río de ferrerías en vasco) se encuentra Legazpi, una acogedora localidad vasca donde han sabido conservar, poner en valor y convertir el patrimonio y el propio municipio en un Museo Vivo, donde se muestra la historia del País Vasco desde el siglo xi hasta 
nuestros días, tomando como hilo conductor el hierro, elemento que ha dado carácter e identidad a sus gentes.

El Valle del Hierro es un lugar donde aún hoy laten las ferrerías y la tradición vasca se palpa en cada rincón, donde viajarás en el tiempo descubriendo la tradición, del mundo rural y artesanal del hierro, los caseríos, la mecanización y la era industrial.

Visitar el valle es sin duda una de las mejores maneras de conocer el País Vasco y sus gentes laboriosas, de entender mejor nuestra cultura y amor por la naturaleza. En tan sólo $5 \mathrm{~km}$, y pudiendo hacer todos los recorridos a pie o en bicicleta, se pueden visitar tanto caseríos con más de 500 años de historia y que todavía hoy siguen elaborando riquísimos productos autóctonos, como el delicioso queso Idiazabal, o el pan artesanal; hasta ferrerías que activan toda su maquinaria dejando boquiabiertos a los visitantes con su fuerza, su ruido y su luz, hasta llegar a la modernidad de la mano de los miles de obreros que llegaron a trabajar al País Vasco buscando un futuro mejor, o Chillida-lantoki, el último de los espacios que abrió sus puertas con la intención de mostrar la relación entre el Arte y la Industria.

Legazpi es un municipio que ha estado vinculado a la actividad industrial, y particularmente al hierro, desde el siglo xi hasta nuestros días.

El municipio está dividido en dos zonas Turístico-Culturales, los barrios rurales y el casco urbano a tan solo dos kilómetros de distancia. En el centro de ambos está el famoso Parque de Mirandaola, un lugar de ocio, donde el visitante encontrará todos los servicios necesarios: Oficina de Turismo (mirandaola@lenbur.com, 943730428 www.lenbur.com), Museos y espacios culturales, áreas recreativas y merenderos, parking de autocaravanas, etc. Este a su vez es el lugar desde donde parten rutas como: la ruta de las Ferrerías, la ruta Obrera u otras supracomarcales como la ruta del Queso, entre otras.

La ruta de las Ferrerías es una sencilla ruta junto al río, desde donde se accede a los barrios rurales de Brinkola y Telleriarte, donde cuidados caseríos vascos salpican el paisaje de los verdes prados. En esta ruta, el visitante podrá ver y comprender el porqué de muchas cosas descubriendo elegantes casas torre, viviendas de los acaudalados dueños de las ferrerías, presas, canales, bosques de hayas trasmochadas (con cuya madera se obtenía el carbón para las ferrerías), que mediante una adecuada interpretación del paisaje nos muestran la realidad del siglo xvi, cuando el hierro vasco se vendía en Europa y América, y donde prácticamente todo el valle vivía del hierro, mineros, carboneros, carreteros, mercaderes, incluso los caseros estaban íntimamente ligados al hierro ya que en muchas ocasiones eran los caseros quienes trabajaban en las ferrerías en invierno, cuando el caudal de agua era abundante para trabajar y cuando en el caserío no había mucho trabajo. Ambas comunidades se necesitaban, unos producían alimentos y los otros herramientas para sus cultivos, pero también eran enemigos, ya que ambos necesitaban el escaso terreno disponible, los primeros para pastos o cultivos y los segundos para producir carbón, el necesario combustible de las ferrerías. Esta fue sin duda una época dorada, donde el «label» del hierro hizo famoso el País Vasco. Pero en el siglo XviII, las tan fructíferas ferrerías hidráulicas ${ }^{1}$

El aprovechamiento del agua como fuente de energía se iba conseguir gracias a un artilugio mecánico llamado rueda hidráulica. Consistía en una gran rueda construida en principio de madera... que llevaba en su circunferencia una especie de palas. Si se colocaba la rueda en el río de forma que el agua chocara con las palas, se conseguía un movimiento giratorio en el eje de la rueda. vv. AA. (1980) Ferrerías en Legazpi (p. 63). Donostia: Caja de Ahorros Provincial de Gipuzkoa. 
sufrieron un duro y terminal golpe, cuando en Inglaterra comenzó la moderna siderurgia, los Altos Hornos entre otros, y donde producían en un solo día lo que en Legazpi producían en un año. La indiscutible ley del mercado hizo que poco a poco todas las ferrerías vascas cerraran, la última en Gipuzkoa fue también en Legazpi, Bengolea (vv. AA., I980: II6), y con ella los cientos de años de glorioso esplendor.

La crisis fue arrolladora, la falta de empleo y de sustento hizo que miles de vascos cogieran barcos hacia las Américas, donde buscaron fortuna como pastores, comerciantes, etc. Otros muchos optaron por el celibato, muy bien visto por las familias vascas y lo que hacía que por lo menos hubiera un comensal menos a comer en la mesa, y otros muchos optaron por el emprendizaje, el del aprendizaje, el de ir a conocer otros países, otras formas de trabajar. Estos vascos, inquietos y valientes, descubrieron nuevas tecnologías que fácilmente se podían adaptar a la caduca maquinaria de las ferrerías. Así surgieron serrerías, en las cuales se utilizaban las mismas ruedas hidráulicas para mover grandes sierras, y que aprovechaban la madera de los árboles que ya no se utilizaban para hacer carbón. También se transformaron en molinos harineros (uno de estos molinos se puede ver todavía en funcionamiento, con más de 500 años de historia) $)^{2}$.

Descubrieron también nuevos materiales, como el papel, un material que se producía artesanalmente en molinos papeleros ${ }^{3}$, el que fueron más tarde uno de los ejes de la industrialización en Gipuzkoa. Llega la industrialización y con ella los pueblos cambian, el paisaje se transforma, las ferrerías se transforman en fábricas y los ferrones en obreros metalúrgicos, comienza una nueva era, la Revolución Industrial.

Y una vez más es en Legazpi donde mostramos esta historia: «La villa había conocido un expectacular crecimiento basado en la producción industrial. Había sido dotada, siguiendo los impulsos de un paternalismo bienintencionado -que no acaba, evidentemente, con los conflictos de clase inherentes a tales sistemas productivos-, de casas para obreros, chalés para ingenieros, escuelas, servicios médicos, etc.».

Por tanto y pedagógicamente hablando haremos un salto desde las ferrerías a las fábricas, desde los barrios rurales a los barrios obreros, desde los ferrones a los obreros metalúrgicos, comprendiendo el porqué de las dos sociedades y por qué una desaparece dando origen a la otra.

La memoria de ese pasado es un legado que define y caracteriza a los habitantes de esta comunidad. Legazpi es el reflejo de muchos pueblos del País Vasco, en los que la industrialización modificó sus hábitos y costumbres, su paisaje, su organización social, las relaciones personales, etc.

En un mundo cada vez más globalizado, hemos creado un discurso identitario, con rasgos propios, pero no localista, porque es aquí, en el Valle del Hierro-Legazpi, donde contamos esta apasionante historia que no te puedes perder.

vv. AA. Brinkola Gogoan, apunte historiko eta etnografikoak, p. 156.

3 En Legazpi se encuentra el conjunto de molinos papeleros mejor conservado de Gipuzkoa, Azpikoetxe, que en el 2009 consiguió entrar en la categoría de Monumento Cultural. Uno de estos molinos papeleros puede visitarse en Papelera-Legazpi, uno de los espacios visitables del Valle del Hierro (www. lenbur.com).

4 Díaz Balerdi, Iñaki, Archipielagos imaginarios. Museos de la Comunidad Autónoma del País Vasco, p. 39 . 


\section{Breve historia del proyecto}

La fundación nació con el objetivo principal de poner en valor el Patrimonio Natural y Cultural y la Industria del Hierro, como un símbolo de la identidad cultural de un pueblo y como un elemento de proyección hacia el exterior, contribuyendo así al desarrollo económico y social.

Este patrimonio estaba desapareciendo rápidamente ante nuestros ojos (las fábricas se desmantelaban, las presas se demolían, los caseríos se abandonaban...). El Ayuntamiento, ante la imposibilidad de gestionar un proyecto que se hacía cada vez más amplio, en el año 1997, decidió crear una fundación sin ánimo de lucro. El nombre de la fundación LENBUR significa LE, Legazpi, que a su vez representa no sólo a un territorio sino también a la propia comunidad, al ser humano que durante siglos ha habitado esta tierra. La $\mathrm{N}$ de Naturaleza, con mayúsculas ya que el majestuoso paisaje humanizado del valle es en sí mismo un lugar magnifico para la interpretación. Y, finalmente, BUR, que significa Burdina, hierro en vasco, y que es el hilo conductor del discurso.

Desde el principio se vio la necesidad de que el proyecto no estuviera únicamente sustentado por el Ayuntamiento, si bien este se comprometió a ser uno de los Patronos y por tanto patrocinadores del ambicioso proyecto, ya que el más beneficiado era el propio municipio. En la fundación se aunaron esfuerzos, haciendo partícipes del proyecto a las empresas como Corporación Patricio Echeverria, s.A., Cie Automotive S.A., GKN Driveline-Legazpi, Urola, Zorrotz, entre otras. También se buscaron figuras relevantes como Eduardo Chillida, quien realizó la gran mayoría de esculturas de hierro de gran tamaño en Legazpi y quien apoyó a la fundación desde sus inicios. Desde el princio se colabora con el INEM en la formación y creación de empleo, con importantes medios de comunicación como el Diario Vasco, entre otros. Más tarde se incorporarán las empresas: Goiber, Construcciones Metálicas Urretxu, U.z. S. Coop., Teledonosti y Punto Radio, zUM, Soroa Arquitectos, EKeI, fundaciones como la Fundación Inasmet y Asociación de Comerciantes Ilinti.

La sensibilización de la propia sociedad era un reto importante, ya que para cuidar y valorar el patrimonio antes hay que conocerlo. Por tanto, llevamos a cabo sendas campañas de sensibilización con diferentes colectivos: escolares, con los que se llevan a cabo diferentes proyectoss. La investigación tenía que ir en paralelo al trabajo de sensibilización ya que el proyecto tenía que ser riguroso en la historia. La fundación impulsó trabajos de investigación ${ }^{6}$. Así mismo, dichos trabajos permitieron a LENBUR Fundazioa hacer acopio de instalaciones, maquinaria, testimonios, mobiliario, planos, fotos, archivos de empresa, cuadros y documentación que ha dado lugar a la creación del Centro de Documentación y Biblioteca Técnica Lenbur, importante referente de investigación y conocimiento del Patrimonio Industrial y de la Memoria del Trabajo en Gipuzkoa: 900 monografías, más de 220 revistas especializadas y más de ro.00o fotografías. Tenemos un archivo especializado que

Las iniciativas son variadas, entre las que se encuentran: concurso estatal de fotografía y pintura, donde el Patrimonio Industrial fuera el tema principal, sendas exposiciones donde el objetivo principal era el acercar la cultura local y el patrimonio a la ciudadanía.

625 investigadores, 20 becas, se han editado o ayudado a editar más de una docena de libros. Se ha recuperado también Patrimonio Tangible, más de 5.000 objetos industriales y de la vida cotidiana, y Patrimonio Intangible, con el proyecto de recuperación de la Memoria del Trabajo en Gipuzkoa. 
cuenta ya con más de 10.000 referencias sobre ferrerías y 2.600 registros sobre la industrialización y el patrimonio industrial. Con más de 6.000 objetos recuperados hoy podemos mantener vivo el testimonio de cómo era nuestra vida cotidiana en el hogar, en el trabajo, en la calle, en la escuela, etc., durante la industrialización.

Desde el principio en la fundación tuvimos claro que la historia debía estar contextualizada, y debía mostrarse en los propios espacios donde había sucedido: caseríos, ferrerías, molinos, fábricas, escuelas, viviendas, etc. Por tanto, otro de los trabajos fue buscar, identificar y recuperar los espacios mejor conservados para posteriormente recuperarlos y musealizarlos. De esta manera se ha dado lugar a la consecución a más de io espacios visitables:

- Ferrería Mirandaola.

- Capilla de la Santa Cruz de Hierro.

- Museo del Hierro Vasco.

- El Rincón del Pan.

- Molino Igaralde.

- Ekomuseo del Pastoreo.

- Aikur. Museo de las abejas.

- Vivienda Obrera, un día en los años 50.

- La Capilla.

- La escuela de 1950, la Escuela Franquista.

- Espacio Chillida.

- Ruta «El latido de las Ferrerías».

- Ruta Obrera.

- Ruta de la Cultura Industrial por Gipuzkoa.

- Oficina de Turismo en Mirandaola.

- Tienda.

- Bar-Restaurante.

- Centro de Documentación y Biblioteca Técnica.

- Sala de Conferencias en el Palacio Bikuña.

Legazpi-El Valle del Hierro es por tanto un Museo Territorio. Un proyecto que ha aunado voluntades públicas y privadas y nos ha posicionado en foros a nivel del País Vasco, estatal e internacional, con más de is proyectos europeos a nuestras espaldas ${ }^{7}$. Y premios y galardones importantes ${ }^{8}$.

- Resider (1995-96-97-98).

- Fondo Social Europeo (1994-95-96-97-98-99).

- Feder (1997-98-99-2000-OI-02-03).

- Raphael (1998-99).

- Cultura 2000 (200I-02).

- Euskadi Aquitania (1998-99-2000).

- Cooperación Transfronteriza e Interregional del Gobierno Vasco (200I-02-04).

- EREIN (2002-06).

- Programa Operativo de Cooperación Territorial España-Francia-Andorra 2007-2013. Ruta del Hierro en los Pirineos-PiriFerro.

8 En estos años de andadura han sido muchos los reconocimientos, entre los que destacan. Habitat II, por las Naciones Unidas (1998).

- Premio Turismo Vasco, por el Gobierno Vasco (2002).

- Mención de Honor, por el Consejo de Europa (2004). 
Pero sin lugar a dudas nuestro mayor mérito es que la Fundación LENBuR ha posibilitado que anualmente visiten Legazpi más de 24.000 personas, cuando hasta hace muy pocos años no era un destino turístico, y no nos visitaba nadie. Esto ha posibilitado un empleo directo de más de 325 personas/contrato/año (1997-2015). Y ha repercutido en la economía local, impulsando proyectos privados dentro del sector turístico, antes no había ni un solo hotel, y actualmente contamos con un hotel urbano, un agroturismo, un camping y unos apartamentos turísticos, entre otros.

\section{Un paseo por el Valle del Hierro}

Recomendamos planificar la visita con tiempo y poniéndose en contacto previamente con la Oficina de Turismo de Mirandaola (www.lenbur.com, mirandaola@ lenbur.com. Tel: 943 730428, Barrio de Telleriarte, s/n. Legazpi-Gipuzkoa) donde le darán las mejores opciones para organizar su viaje, ya que en el Valle del Hierro tenemos actividades diferentes cada estación del año y adaptamos la visita a las características del visitante, ya que tenemos ofertas para todos los gustos: escolares, planes en familia, para grupos, de senderismo, etc. Será también en la Oficina donde obtendréis todas los horarios y entradas necesarias para realizar una visita optima.

El Parque de Mirandaola será el punto de partida para conocer esta apasionante historia.

En las mismas instalaciones donde se encuentra la Oficina se encuentra también el Museo del Hierro Vasco, y mediante una visita guíada, de una forma amena y muy didáctica, nos darán las claves para entender la importancia del hierro en el País Vasco y nos mostrarán aspectos tecnológicos, históricos, científicos y sociales que han hecho evolucionar al propio territorio vasco y, cómo no, también a sus gentes.

A escasos Ioo $\mathrm{m}$ y atravesando los pequeños puentecillos del parque, descubrimos. la Ferrería de Mirandaola, es uno de los elementos patrimoniales más importantes de Gipuzkoa. Los ferrones vestidos con sus largos vestidos de lino y sus sombreros de ala ancha nos invitarán a pasar a su interior, donde, una vez dentro, nuestra mente se trasladará al pasado situándonos en el siglo xvi: el sonido del agua al caer fuertemente sobre las palas de la gigantesca rueda hidráulica que hace que se muevan los enormes fuelles, el gran horno donde se consiguen más de $1300{ }^{\circ} \mathrm{C}$ para calentar la pieza de hierro. El martillo, de más de tres metros de largo, golpea el metal al rojo vivo produciendo un ruido ensordecedor. Todo ello nos hace testigos de una experiencia única en todo el País Vasco, el del duro trabajo que los ferrones hacían en este lugar. Serán los propios ferrones los que además de realizar el espectáculo nos cuenten mil y una interesantes historias, entre las que se encuentra ¡el milagro! que acaeció el 3 de mayo de 1580 en este mismo lugar y por el que siglos después se construyó la Capilla. Uno de los secretos mejor guardados del valle y que descubriréis en esta visita?.

\footnotetext{
- Premio Montblanc (2007) (Premio en metálico).

Q de Calidad Turística (2007).

- Mención de Premis Cat Turismo (2007).

- Bai Euskarari Ziurtagiria. Zerbitzua Euskaraz (2007-2008).

- Protagonista del año, por Punto Radio, en la categoría de Turismo Cultural (2008).

9 El Milagro de la Cruz de Hierro. Era mayo de I580, un invierno seco hacía que el caudal del río fuera cada vez más escaso y el administrador de la ferrería, para poder hacer frente a los pedidos de hierro que tenían apalabrados, decidió que la ferrería no paraba el domingo, el único día de la semana que cerraba
} 
La visita en la ferrería finalizará con el tradicional «Pintxo Ferrón»: chistorra recién hecha en las brasas de la ferrería con pan de nuestro caserío ${ }^{\text {To }}$ regado con sidra natural, una pequeña fiesta gastronómica con algunos de los productos más ricos y típicos.

Desde el parque seguiremos las señales que nos indican el camino de la Ruta de las Ferrerías, donde la primera vivienda a escasos 500 metros es un caserío reconvertido en un precioso Agroturismo ${ }^{\text {II }}$ con acogedoras habitaciones con visitas al Parque Natural del Aizkorri. Siguiendo el camino, descubriremos los tesoros de la ruta: las casas torre, ermitas, presas, canales e incluso podremos visitar alguno de los caseríos vascos mejor conservados, que nos abren las puertas de su casa y nos muestran sus oficios ${ }^{12}$.

Uno de estos caseríos es Erreizabal, rodeado de verdes pastos para el rebaño de ovejas Latxas ${ }^{13}$. En el mismo caserío encontraremos el Ecomuseo del Pastor, a través del cual podremos conocer el trabajo del pastoreo desde dentro, la producción del queso Idiazabal y una pequeña tienda donde se puede adquirir este típico queso vasco producido en la propia quesería. Juan Jose Aranguren, pastor, y Arantza Segurola, quesera, nos recibirán con la hospitalidad propia de buenos vascos, y nos mostraran los mil y un secretos de la Oveja Latxa, muchas veces premiada nacional e internacionalmente por la elaboración de sus quesos, un manjar para los sentidos, les mostrarán todos sus secretos. Si lo desean también ofrecen el pintxo pastor con queso y pan de caserío.

Qué mejor acompañamiento para el queso que una buena hogaza de pan reciente hecho en horno de leña, como se hacían antes las cosas, o como se diría ahora «slow food», dejando reposar la masa toda la noche. A escasos 500 metros llegaremos al Caserio Igaralde, un lugar que no te puedes perder si eres amante del buen pan. Tibur Agirre, quien además de enseñarnos el horno antiguo, el moderno y el Museo del Pan, también nos pondrá en funcionamiento un molino de más de 500 años de historia. Este es un lugar donde conocer el origen, la tradición y la historia del pan: el trabajo de la tierra, las herramientas y su evolución, el cultivo, la semilla, separar el salvado de la harina, la masa y finalmente el pan, sin conservantes ni colorantes.

Los anfitriones son muy importantes para nosotras, ya que son los ferrones, los caseros, queseras, pastores y molineros los que nos abren las puertas de su casa y

\footnotetext{
la ferrería para acudir a la iglesia. Esa mañana los ferrones introdujeron, como era habitual, I4 cargas de carbón vegetal y el mineral en el horno, suficiente como para sacar aproximadamente 55 kilos de hierro limpio. Aumentaron la temperatura hasta $\mathrm{I} 300^{\circ} \mathrm{C}$ y esperaron siete horas como acostumbraban. A la hora de sacar la pieza del horno se encontraron con una pequeña pieza en forma de cruz, que solamente pesaba 5 kilos. Los ferrones, asustados, escondieron la cruz y fueron a misa, manteniendo el secreto de la Cruz de Mirandaola de padres a hijos durante generaciones, hasta que un obispo de Navarra decidió investigar el tema y la comunidad encontró la cruz que hoy día se encuentra en la Parroquia de la Asunción de Legazpi, y que se lleva en procesión hasta la ermita de Mirandaola el 3 de mayo, que a su vez se ha convertido en la Fiesta Grande para todos y todas las legazpiarras.

Io Igaralde Baserria-El Rincón del Pan. El único caserío del valle que conserva el molino, el horno antiguo y que todavía hoy elabora y vende pan artesanal.

II Agroturismo Pastain, Barrio de Telleriarte. Legazpi.

${ }_{12}$ Indispensable reservar hora en la Oficina de Turismo de Mirandaola.

${ }^{13}$ Es la raza autóctona y debe su nombre a la palabra «latza», que en euskara significa áspero, palabra que hace referencia a la lana que la protege de los duros inviernos, pero que produce más de dos litros de leche al día. El pastoreo ha sido uno de los ejes de la economía de la zona en el r. ${ }^{\circ}$ sector. En Legazpi hay dos queserías tradicionales, un Museo del Pastor, la Cofradía de la Oveja y se celebra el Artzai Eguna de Gipzukoa todos los años el primer domingo de septiembre.
} 
nos muestran su saber hacer, convirtiendo la visita en una experiencia inolvidable. Antiguamente en los caseríos los abuelos contaban a los nietos infinidad de historias, pasando su conocimiento de generación en generación. Ahora son los caseros-as quienes se encargan de difundir ese saber familiar a los visitantes que acuden a su casa.

Y centrándonos en sus gentes damos comienzo a la siguiente parte de esta propuesta, durante el último siglo en el País Vasco, miles de trabajadores llegaron buscando un futuro mejor para ellos y sus familias. La vida del valle se transforma de la vida rural y artesanal a la industrial. Donde el ferrón se convierte en el obrero; la ferrería en fábrica; el baserri (caserío) en vivienda obrera, etc. Nos adentraremos en el mundo de familias obreras, descubriremos en más de is espacios reales ${ }^{14}$, para descubrir cómo llegaron y cómo vivieron, la historia de la vida cotidiana del último siglo en Euskadi se cuenta en este apasionante relato.

La fábrica es el punto de partida de estos apasionantes espacios a descubrir, donde veremos cómo es una antigua fábrica, ficharemos, tomaremos las pertinentes medidas de seguridad, descubriremos por qué se produjo el cambio de una sociedad rural a una sociedad industrial. El obrero, vestido con el buzo azul de mahón, es el protagonista de esta historia. Las duras condiciones laborales, las largas jornadas, la prohibición de sindicatos u organizaciones de trabajadores, entre otras, son algunas de las duras historias que nos mostrarán. Pero también se hará hincapié en los empresarios, emprendedores, los cuales empezaron a forjar pequeñas industrias en el territorio, con poco capital, pero con muchas ideas y mucha ilusión y que poco a poco, con gran esfuerzo, inteligencia y saber hacer, consiguieron que Gipuzkoa y el País Vasco fueran, un lugar próspero, donde había trabajo para todos, un lugar de acogida para miles de familias, y donde se fraguaron los mimbres de la sociedad del Bienestar en la que hoy nos encontramos. Por tanto, la Fábrica es un homenaje a todos esos hombres, obreros y empresarios, que trabajaron en cuerpo y alma para sacar adelante a sus empresas, sus familias, etc.

Dentro de Papelera-Legazpi, se encuentra también Chillida Lantoki, un espacio único, un antiguo edificio industrial reconvertido en Museo, y el cual ha recibido numerosos premios de arquitectura; en él, se muestra la relación entre el Arte y la Industria, de la mano de uno de los escultores más famosos del País Vasco, Eduardo Chillida, quien vino a Legazpi-el Valle del Hierro a realizar sus esculturas monumentales que hoy están en plazas y museos de todo el mundo. En este espacio se muestran los talleres de papel y de forja del escultor, las máquinas de la Gran Forja, modernos sistemas audiovisuales y entrevistas a los trabajadores, entre otros. Estas esculturas no se hubieran podido realizar sin la ayuda que Patricio Echeverría dio a Chillida en sus comienzos, cuando ambos, trabajo técnico y capital humano en la fábrica y capacidad de creación artística del escultor, realizaron la primera escultura El Peine del Viento-Donostia, y que fue el comienzo de un largo viaje, ya que las esculturas de hierro que hoy día se encuentran en plazas y museos de todo el mundo están hechas en Legazpi en el Valle del Hierro con la colaboración de los ferrones del siglo xx, los obreros metalúrgicos.

Al salir de la fábrica realizaremos el mismo recorrido que hicieron tantos y tantos obreros, donde además podremos visitar espacios recreados in situ, como la vivienda

${ }^{14}$ Economato, dispensario médico, casas de ingenieros, hotel del la empresa, casa del amo, barrios obreros, entre otros. 
obrera $^{15}$, donde las familias vivieron en pisos compartidos, ya que obtener una vivienda fue un problema gravísimo. Las familias se organizaron y se dieron diferentes fórmulas de convivencia, vecinos con derecho a cocina, pupilos, hospederías, incluso en algunos casos hubo familias que se vieron obligadas a alquilar bordas de ganado. La Vivienda ${ }^{16}$ es un espacio magníficamente reconstruido, donde la cocina es el eje de la vida familiar y donde la mujer es la protagonista, administradora, maestra, cuidadora, costurera y poseedora de mil oficios más a los que vulgarmente se les llamó «mis labores». En la vivienda retrocederemos en el tiempo hasta llegar a los duros años del franquismo, en plena postguerra, donde el hambre y la escasez eran el pan de cada día.

La fábrica también se encargó de abastecer de alimentos a sus obreros, creando el Economato Obrero, un supermercado un tanto especial donde sólo se podían adquirir bienes de primera necesidad y racionados, pero donde los precios eran mucho más asequibles que en el mercado libre. Las transacciones en el Economato se realizaban mediante las «cartillas», y los recuerdos comunitarios de este establecimiento son las largas horas de espera que realizaban la mujeres para obtener entre otros productos sebo para hacer jabón o menudencias. La sanidad fue suplida también por la empresa, quien construyó un Hospital y un dispensario médico antituberculoso, donde los obreros y sus hijos pasaban chequeos médicos anuales, y una de las primeras iniciativas en concepto de prevención que se dio en todo el País Vasco.

La Educación tuvo, cómo no, un peso fundamental e importantísimo en todo el proceso de adoctrinamiento de las clases obreras y en el caso de Legazpi fue el primer edificio de carácter social que construye la empresa, en 1943. El objetivo principal era la educación de los hijos varones de los obreros, es decir, asegurarse de un relevo generacional, adoctrinado, dócil y fiel a la empresa ${ }^{17}$.

La educación de las chicas no interesaba demasiado y no fue hasta 25 años más tarde, y después de una gran presión social, cuando Patricio decide construir el colegio de las chicas, el Colegio de Santa Teresa (en honor a su esposa).

El mundo educativo de esos años, el Nacional Catolicismo y todo lo que ello supuso para muchas generaciones, que recuerdan como si fuera hoy todos los detalles de la escuela, el retrato de Franco en la pared, el crucifijo, los pizarrines, pupitres y enciclopedias... cada detalle ocupa su lugar en riguroso orden y donde la rigidez, disciplina y religiosidad eran los principales valores y donde los niños y las niñas estudiaban en colegios diferentes y cada uno era educado para cumplir su «función» en la sociedad. Tú puedes ser el protagonista de esta película en blanco y negro donde levantando cada pupitre descubrirás mil historias.

La historia que contamos en Legazpi no es una historia local, es un ejemplo de los iconos en la industrialización gipuzkoana. Pero Legazpi es mucho más, en sus cuidadas calles, podréis disfrutar de un buen pincho o de una exquisita comida en los muchos restaurantes, que trabajan con productos locales. Y si queréis también podéis

is La empresa durante un largo periodo de tiempo suplió la función municipal de ofrecer vivienda a los trabajadores. Al fin y al cabo era el crecimiento de la propia empresa el que provocaba la llegada de una masiva población foránea a la que había que alojar (Herreras y ZALDUA, 1997: 87).

${ }_{16}$ Ubicada en el primer Barrio Obrero será San Ignacio, y es aquí, en el n. ${ }^{\circ} 5$, donde Lenbur adquirió una vivienda y la ha recuperado tal y como era.

${ }^{17}$ En las entrevistas orales realizadas referentes a este tema en más de una ocasión se mencionaba «Patricio Echeverría no necesitaba intelectuales, solo quería obreros sumisos». 
probar una de las especialidades del valle, el Ferron Gin, un gin tonic inspirado en la ferrería de Mirandaola.

\section{Bibliografía}

Aranburu, Aintxine y Etxeberria, Birginia (2005) Burdinlariak, Oficios relacionados con la producción artesanal del hierro. Fundación LENBUR.

Aranburu, Aintxine y Etxeberria, Birginia (2007) Burdinlariak, Zeharrolas, Ferrerias en el Alto Urola. Fundación LENBur.

Ayerbe, M. ${ }^{a}$ Rosa (1995) Fuentes documentales medievales del País Vasco. Documentación medieval del archivo municipal de Legazpi. Eusko Ikazkuntza.

Candela, Paloma; Castillo, Juan José y López García, Mercedes. Arqueología Industrial y memoria del trabajo: patrimonio industrial del sudeste de Madrileño, I905-195o.

CASTILlo, Juan José (2008) La soledad del trabajador globalizado, memoria, presente y futuro.

FERnÁndez Gómez, Julio A. Buscando el pan del Trabajo, sobre la industralización franquista $y$ sus costes sociales (1940-1965).

Herreras, Beatriz y Zaldua, Josune (1997) Patrimonio Industrial en Legazpi (p. 87). Legazpi: Fundación LENBUR.

Legorburu, Elena (2004) La labranza del Hierro en el País Vasco, hornos, ruedas y otros ingenios. Servicio editorial de la Universidad del País Vasco.

vv. AA. Brinkola Gogoan, apunte historiko eta etnografikoak. pp. 156

vv. AA. Ferrerías en Legazpi (I980), Donostia: Caja de Ahorros Provincial de Gipuzkoa.

vv. AA. Legazpi (1980) Ayuntamiento de Legazpi. 